\title{
APLICACIONES SOLARES COMO ALTERNATIVA DE AHORRO ENERGÉTICO EN CAMPAMENTOS MINEROS
}

César Rivasplata $C$.

\author{
R E S U M E N
}

Southern Peru Limited (SPL), en sus campamentos mineros de Cuajone y Toquepala, ubicados a 3,000 m.s.n.m. y con una población cercana a las 20,000 habitantes, brinda no sólo facilidades de energía eléctrica, sino también servicios de agua para uso domiciliario, calefacción y recreación, mediante sistemas convencionales que usan petróleo.

Durante los años 1992-1993, la empresa emprendió el desarrollo de un plan piloto a fin de ahorrar petróleo en el calentamiento de agua para uso doméstico, utilizando como fuente de energía alternativa la energía solar.

SPL ha ejecutado un proyecto piloto para el calentamiento de agua de uso domiciliario, instalando colectores solares para cuarenta apartamentos familiares (cinco pabellones de ocho apartamentos familiares de cinco personas/familia), contribuyendo con un ahorro promedio del $30 \%$, de un consumo usual de $600 \mathrm{~g} / \mathrm{s}$. de petróleo/mes/pabellón (incluyendo calefacción).

El otro proyecto realizado ha sido el uso de colectores solares para el calentamiento de agua en las piscinas de Toquepala y Cuajone, a fin de mantener temperaturas confortables, del orden de 28 a $30^{\circ} \mathrm{C}$. Los sistemas de las piscinas se encuentran operando y representan un ahorro promedio de 2,600 gl/petróleo/piscina/año, esperándose un retorno de capital en un tiempo de dos años.

En la presente ponencia se desarrollan los alcances técnicos y económicos logrados mediante el uso de la energía solar como alternativa de ahorro energético en estos dos proyectos.

\section{A B S TR A C T}

Southern Peru Limited (SPL), in its mining camps of Cuajone and Toquepala, wich are situated at 3000 metres above sea level, and have a population of approximately 20,000 inhabitants, provides facilities not only of electical energy, but also of water supplies for domestic use. heating and recreation, through conventional systems which use oil.

During the years 1992-1993, the company began the development of a pilot scheme which had the object of saving oil in the heating of water intended for domestic use, using as an alternative source of energy solar energy.

SPL has carried out a pilot project for the heating of water intended for domestic use. installing solar collectors for forty family apartments (five pavillions of eight family apartments of five people/family). contributing an average saving of $30 \%$, from a usual consumption of 600 gallons of oil/month/pavillion (including heating).

Another project which has been carried out has been the use of solar collectors in the swimming pools at Toquepala and Cuajone, with the object of maintaining a comfortable temperature, of the order of 28 to $30^{\circ} \mathrm{C}$. The systems of the swimming pools are in operation and represent an average saving of 2600 gallons/oil/swimming pool/year, which it is hoped will recuperate the capital invested within two years.

In this article the technical and economic advances made through the use of solar energy as an alternative for saving energy in these two projects are explored. 


\section{ANTECEDENTES}

SPL, a través de sus áreas de Toquepala y Cuajone, ha venido promoviendo el uso de fuentes de energía renovables, básicamente la solar, con fines de ahorro y conservación de energía. Los primeros intentos se remontan al año 1981, cuando en coordinaciones realizadas entre la SPL y la UNJBG de Tacna se absuelve una consulta de probable aplicación de energía solar para la calefacción de una piscina en Toquepala (1).

En 1983 la división de Ingeniería - Cuajone emite un reporte sobre Experimentos de Energía Solar (2), siendo objetivo del mismo demostrar la factibilidad técnico-económica del uso de colectores solares para el calentamiento de agua para uso doméstico.

En 1986 se establece la firma de un convenio (SPLUNJBG) con la finalidad de realizar un estudio de investigación relacionado con la "Utilización de la Energía Solar en Generación Térmica para Vivienda". Este proyecto se realizó durante el año 1987, existiendo información metereológica de Villa Cuajone (3), de relevante importancia en las futuras proyecciones de aplicación de proyectos en esta área. También se realizaron dos reportes (4), donde se detallan las acciones ejecutadas y los logros alcanzados.

Como resultado de estas acciones e inquietudes propias del personal de la empresa, durante los años 1988-1990, se han realizado diversas actividades, desde la construcción de colectores solares, utilizando materiales disponibles en la empresa (supplies), con la finalidad de calentar agua para uso doméstico en algunas casas de Villa Cuajone, así como para formar parte de la calefacción solar de la piscina $(20 \mathrm{~m} \times 10 \mathrm{~m})$ de Villa Cuajone.

En 1991, SPL oficializó el desarrollo del programa "Solar Heating", habiéndose establecido como metas el uso de colectores solares en cinco pabellones de ocho apartamentos cada uno y la calefacción de las piscinas de Cuajone y Toquepala, con el objetivo de ahorrar petróleo para el uso de calentamiento de agua con fines domésticos (5) (6).

\section{ESTUDIOS PRELIMINARES. EVALUACIONES DE CONSUMO Y AHORRO DE ENERGÍA}

El uso de la energía solar está limitado por las fluctuaciones y características propias de la variabilidad diaria y estacional del recurso y porque los sistemas y el estado del arte actual de los equipos convertidores de energía solar es relativamente caro (\$200-\$300 USA $\mathrm{m} 2$ colector) en términos de inversión inicial.

Estas desventajas son superadas si se conceptualiza que la energía solar debe ser vista como ENERGíA DE AHORRO, la misma que debe ser usada en sistemas mixtos (solar-convencional), con el objetivo de satisfacer y optimizar la demanda.

El aspecto del costo de los sistemas solares indica una tendencia a la baja, en la medida que el petróleo incremente sus precios y que los sistemas solares sean difundidos masivamente. Complementariamente, aspectos inherentes al medio ambiente y la contaminación generada por los combustibles, fortalecen la necesidad de un cambio y uso de nuevas fuentes alternativas de energía, lo que ha de repercutir en los costos de producción y de venta al usuario final.

Es importante considerar también las políticas de gobierno en lo que concierne a los incentivos y aspectos tributarios por el uso de energías renovables, como una forma indirecta de subvención en el ahorro de energía y la prevención del medio ambiente, así como el de integrar a poblaciones rurales aisladas que carecen de energía eléctrica y que potencialmente no tendrán esta necesidad por muchos años más, postergando su integración al desarrollo nacional.

En el año 1987, en Cuajone se realizaron estudios preliminares de evaluación de consumo de petróleo para uso domiciliario, en comparación con el uso de la energía solar, ensayándose un sistema mixto solarpetróleo. Las evaluaciones realizadas determinaron los parámetros de radiación solar, rango de temperaturas del agua a la entrada y salida de los colectores del sistema prototipo, así como el tiempo total diario de uso del caldero, habiéndose obtenido una relación interdiaria para un promedio representativo del mes, proyectándose ello a efectos de realizar una evaluación promedio mensual (Fig. $\mathrm{N}^{\circ} 1$ ).

La Fig. $\mathrm{N}^{\circ} 2$ muestra la energia solar diaria, incidente por metro cuadrado. Esta energía solar está disponible para ser captada por los colectores solares.

Basándose en estos resultados, se estimó la energía total cedida al sistema y las contribuciones solar y de petróleo para un día promedio (julio 97), siendo la proporcionalidad de energía consumida, tanto para calefacción y el calentamiento de agua, la siguiente :

\begin{tabular}{|l|r|c|}
\hline ENERGíA & kwh & $\%$ \\
\hline & & \\
Petróleo & 143.8 & 87.4 \\
Solar & 20.7 & 12.6 \\
Total & 164.5 & \\
\hline
\end{tabular}


Siguiendo la misma metodología, se determinó el consumo energético anual para el año 1997, considerando un consumo de petróleo de $80 \mathrm{ml} / \mathrm{min} \mathrm{x}$ tiempo caldero (Fig. № 3) y la energía promedio consumida/día como sigue:

\begin{tabular}{|l|l|l|l|l|}
\hline Mes & $\begin{array}{l}\text { Solar } \\
\text { kwh/día }\end{array}$ & $\begin{array}{l}\text { Petróleo } \\
\mathbf{k w h} / \text { día }\end{array}$ & $\begin{array}{l}\text { Total } \\
\mathbf{k w h} \text { /dia }\end{array}$ & $\begin{array}{c}\% \text { Contribución } \\
\text { solar }\end{array}$ \\
\hline Mayo & 22.7 & 11.2 & 134.9 & 16.8 \\
Junio & 26.5 & 126.6 & 1476.1 & 13.9 \\
Julio & 20.7 & 143.8 & 164.5 & 12.6 \\
Agosto & 24.5 & 123.6 & 148.1 & 16.5 \\
Setiembre & 28.4 & 91.8 & 120.2 & 25.2 \\
Octubre & 29.1 & 86.4 & 115.5 & 25.2 \\
\hline Oct. & $(28.3)$ & 154.0 & 153.0 & $(18.5)$ \\
& & & & sin colector \\
Nov. & $(32.5)$ & 57.6 & 57.6 & $(56.4)$ \\
Dic. & $(34.2)$ & 60.0 & 60.0 & $(57.0)$ \\
\hline
\end{tabular}

FIGURA $N^{\circ} 1$

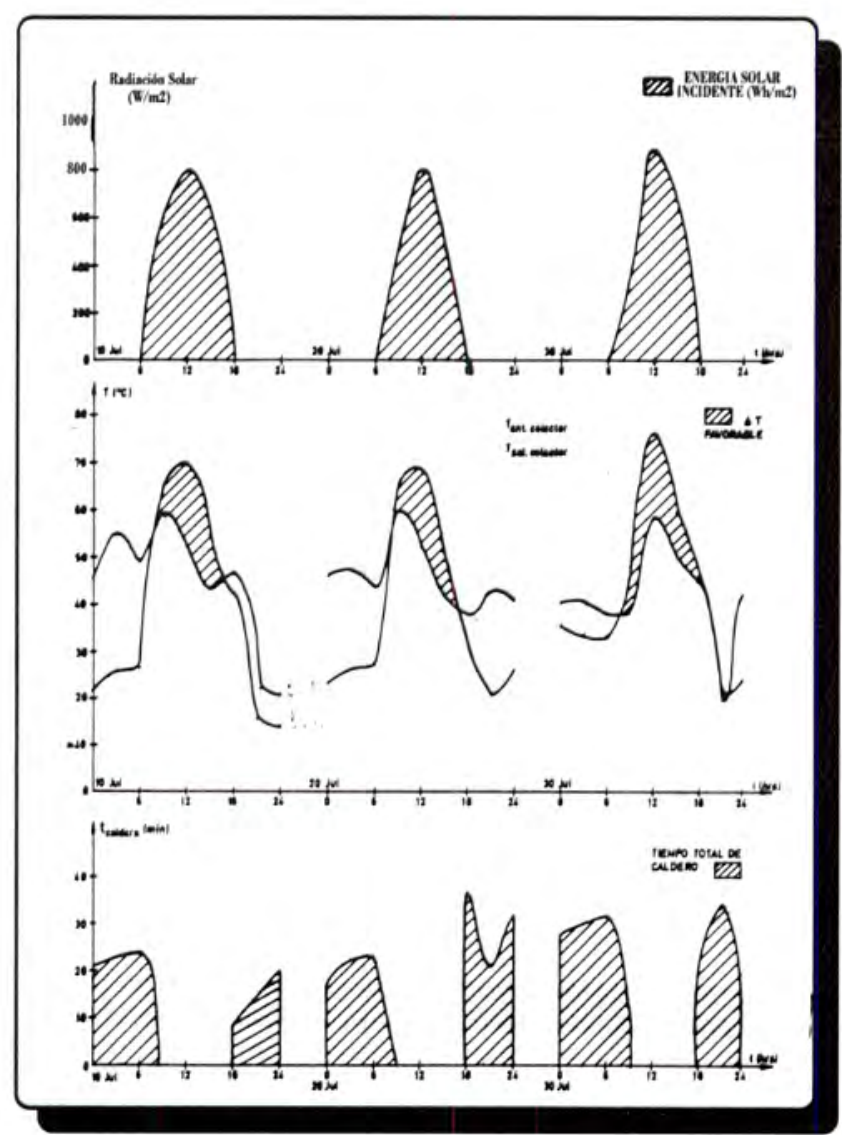

FIGURA $N^{o} 2$

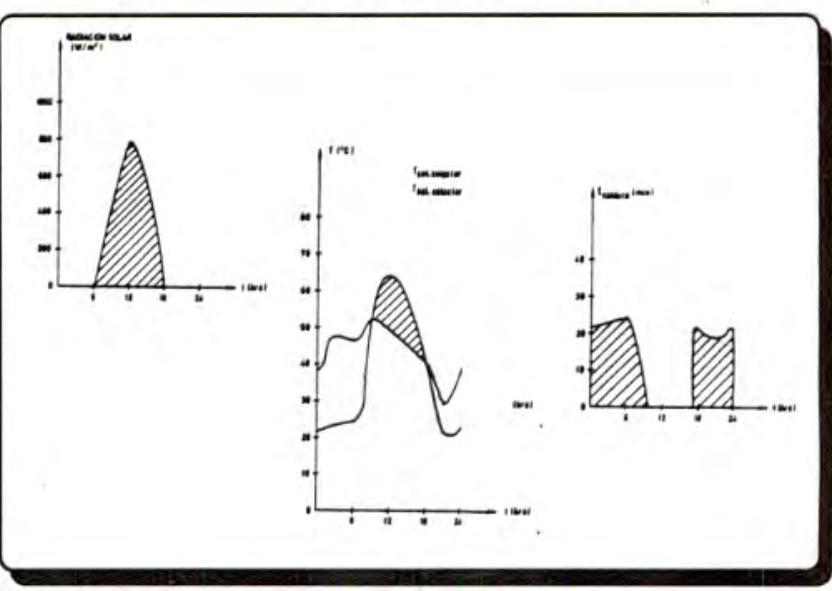

FIGURA $N^{\circ} 3$

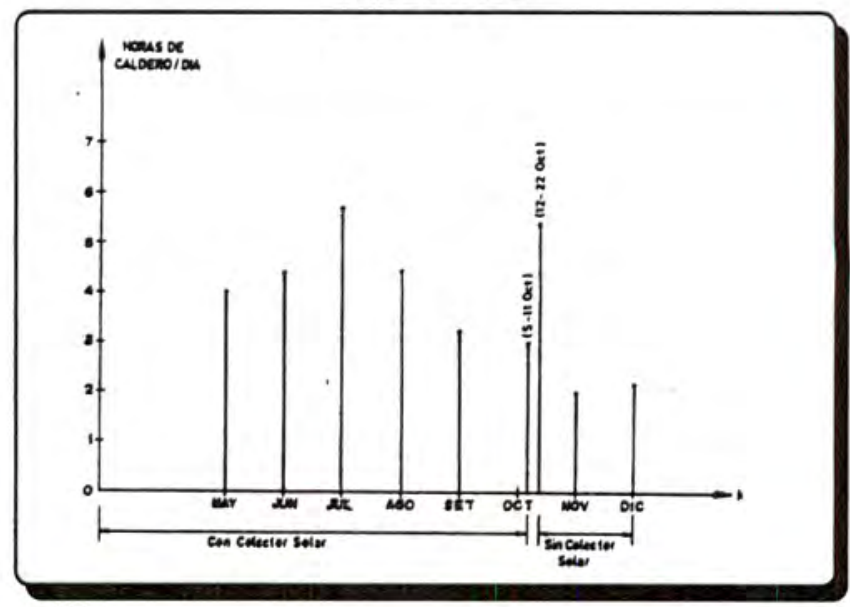

De las Tablas puede apreciarse que para el mes de julio 97 (mes de radiación solar baja, comparada con otros meses) el aporte solar en el consumo de energía total (agua caliente + calefacción) fue del $12.6 \%$, representando ello el $55.4 \%$ en el rubro exclusivo de uso para el calentamiento de agua, siendo este procentaje variable en función del tiempo (Fig. $\mathrm{N}^{\circ} 3$ ).

\section{III.SISTEMA PROTOTIPO DE COLECTORES SOLARES PARA UN PABELLÓN}

En 1991 se instaló el primer sistema de colectores solares en Villa Cuajone (pabellón 317 - 324 Calle Sama), empezando a operar en octubre de 1991 (diagrama sistema hidraúlico).

El sistema fue construido con 14 colectores de $2 \mathrm{~m}^{2}$ de área cada uno, cubriéndose un área de colección solar de $28 \mathrm{~m}^{2}$ con el objetivo de suplir necesidades de agua caliente del orden de $500-600 \mathrm{gl} /$ día a temperatura promedio de $50^{\circ} \mathrm{C}$. 


\section{DESARRollo deL PROYECTO. EVAlua- CIONES. RESULTADOS}

Se realizaron diversas evaluaciones de consumo de agua caliente, consumo de petróleo, temperaturas de entrada y salida del agua de los colectores, con el objetivo de optimizar las instalaciones proyectadas en el resto de los pabellones de las calles Sama y Omate (Villa Cuajone).

Sistemas similares al primero han sido instalados en los pabellones restantes, habiendo empezado su funcionamiento, según las fechas que a continuación se indican :

\begin{tabular}{|l|l|l|l|}
\hline Pabellón & Calle & Lugar & Fecha \\
\hline $301-308$ & Sama & V.C. & $92-03-14$ \\
$309-316$ & Sama & V.C. & $93-03-26$ \\
$341-348$ & Omate & V.C. & $92-04-07$ \\
$333-340$ & Omate & V.C. & $92-04-22$ \\
\hline
\end{tabular}

Los colectores empleados en estos últimos pabellones han sido construidos en los talleres de SPL.

Una parte de los materiales empleados (tubos de cobre, conexiones, elementos de control) han sido material surplus, mientras que otros materiales (planchas de cobre, aislamientos, cubiertas) fueron comprados localmente.
El proceso de construcción integral de 30 colectores, de aproximadamente $3.7 \mathrm{~m}^{2}(1.20 \mathrm{~m} \times 3.1 \mathrm{~m})$, hacen un total de $111 \mathrm{~m}^{2}$ de área de colector y cubren las necesidades de 5 pabellones.

El sistema de colectores instalado sigue el desarrollo del diagrama adjunto (Fig. $\mathrm{N}^{\circ}$ 4). Es un sistema de circulación forzada y los colectores estén distribuidos en paralelo, habiéndose cuidado la distribución del flujo y de carga, al emplearse el concepto de retorno invertido.

El sistema de control funciona en base a un sensor de temperatura, con la condición de que cuando la temperatura ambiente es de $21^{\circ} \mathrm{C}$, se comanda la apertura o cierre de válvulas solenoides que permitan la circulación de agua desde la parte inferior del tanque de almacenamiento hacia los colectores.

\section{EVALUACIONES Y RESULTADOS}

El consumo total típico, de agua caliente para un pabellón de 8 apartamentos con un promedio de 5 personas/apartamento, oscila entre 1000-1200 gl/dia con un consumo promedio de 25-30 gl/pers/día.

El aporte energético solar para el calentamiento de esta agua es de $50 \%$. El consumo promedio mensual de petróleo en el pabellón 317-324 desde octubre 91 a enero 92 fue de $293 \mathrm{gl} / \mathrm{mes}$, mientras que el consumo promedio de los 5 pabellones restantes de las calles Sama y Omate, asciende a $481 \mathrm{gl} / \mathrm{mes}$. En términos comparativos porcentuales, ello representa un $40 \%$ de ahorro de petróleo.

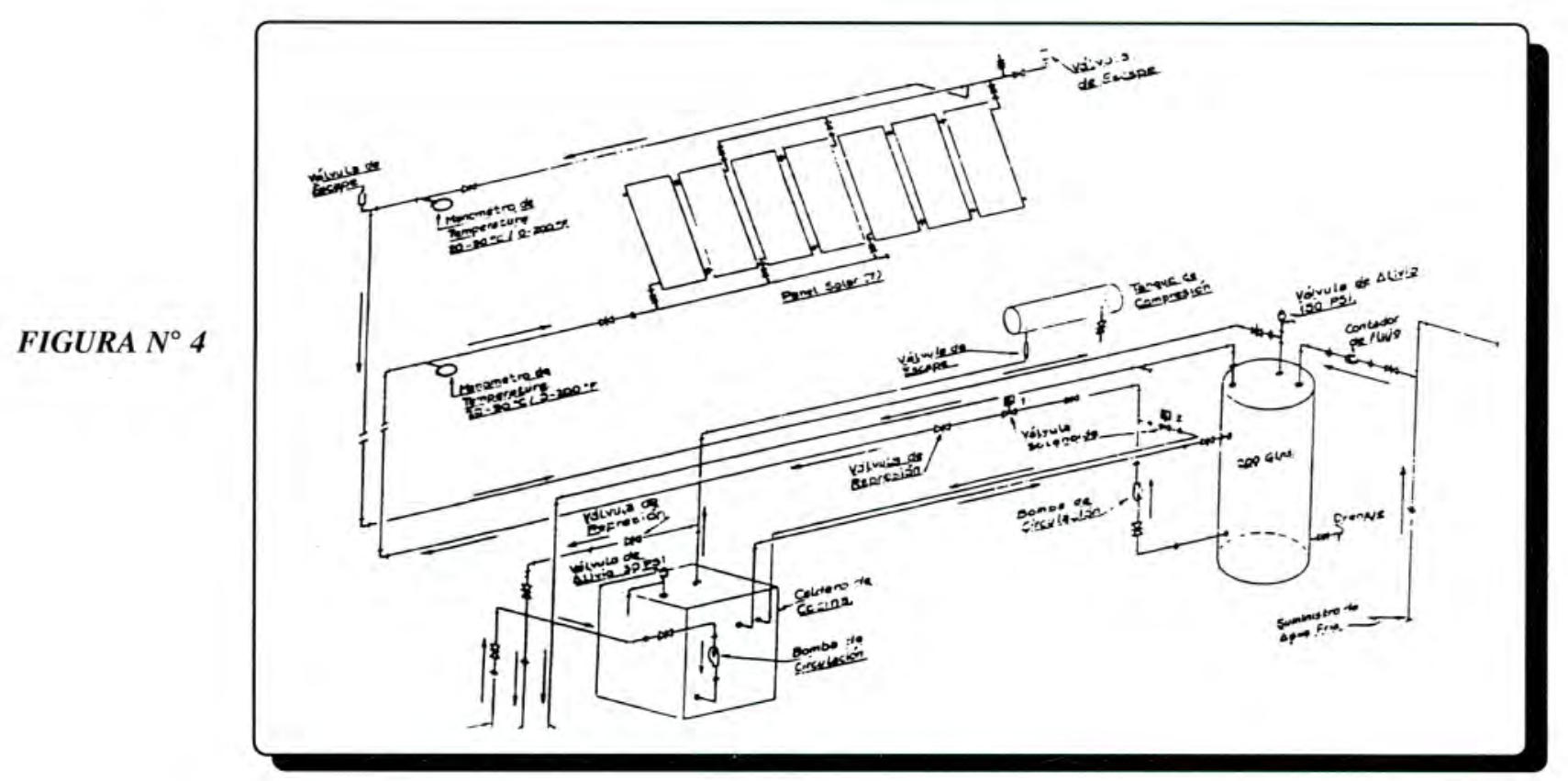


Para el período abril-19/ mayo (42 días), periodo en que la mayoría de sistemas solares estaban en operación, el consumo de petróleo para los 5 pabellones asciende a 2541 galones, lo que representa un promedio de $10.4 \mathrm{gl} /$ pabellón con el consumo promedio desde octubre 91-enero 92 (Gráfico: comparación consumo/petróleo, Fig. Nros. 5, 6 y 7), periodo de menor frío, donde aun el porcentaje de ahorro es del $35 \%$.

Así, considerando un ahorro promedio anual del $30 \%$ para consumo de petróleo en los pabellones $\mathrm{Cl} 301$ 348 , el mismo que fue de 38,759 galones para 1991 , el ahorro anual esperado debió ser 11,627 galones, lo que a precios de esa fecha $(\$ 1,59 / \mathrm{gl})$, equivaldría a un ahorro de $\$ 18,486 / a n ̃ o$.

En términos económicos y de rentabilidad, y asumiendo las siguientes variables :

FIGURA $N^{\circ} 5$

COMPARACIÓN CONSUMO/PETRÓLEO ENTRE 5 PABELLONES SIN E.SOLAR Y 1 CON E.SOLAR

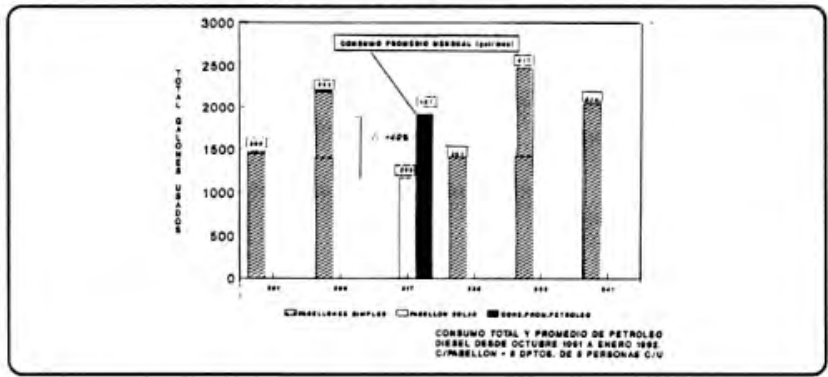

FIGURA $N^{\circ} 6$

CUADRO DE CONSUMO DE COMBUSTIBLE: CALLES SAMA Y OMATE - VILLA CUAJONE

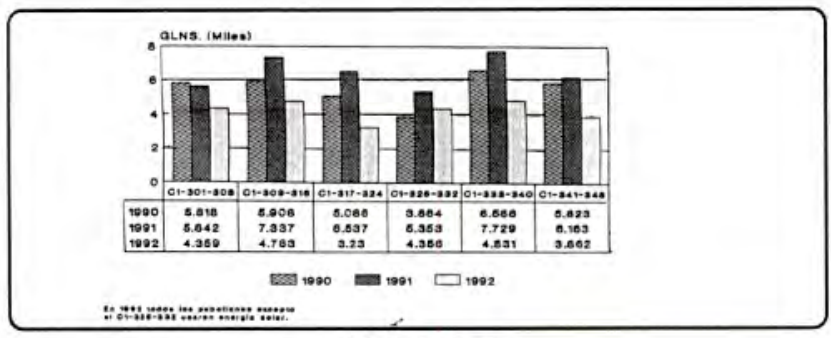

FIGURA $N^{\circ} 7$

CUADRO DE CONSUMO DE COMBUSTIBLE: CALLES SAMA Y OMATE - VILLA CUAJONE

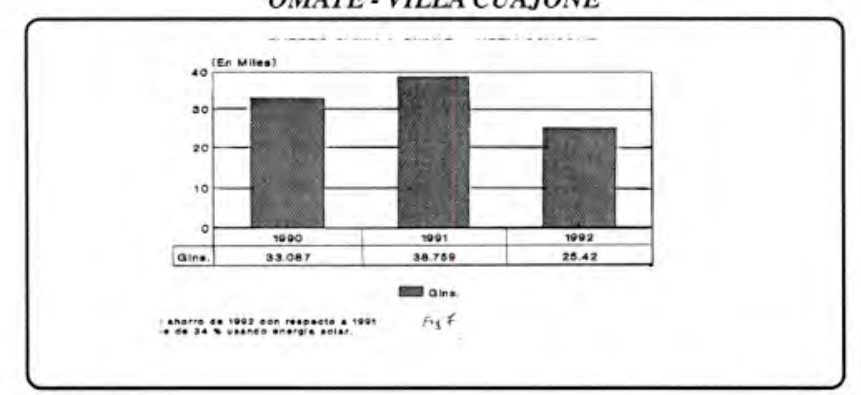

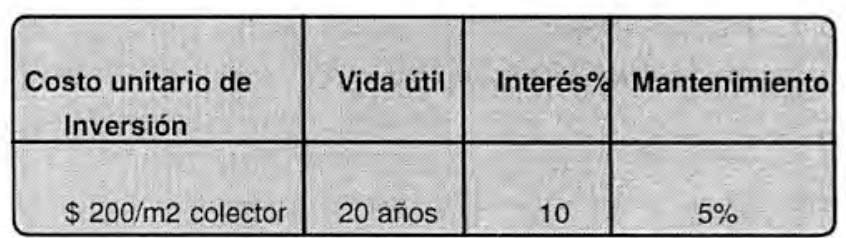

La anualidad es de $\$ 22.4 / \mathrm{m} 2$, sumando un total de $\$ 4,461$; la misma que con un beneficio expresado por el ahorro porcentual de petróleo, tiene un valor crítico de rentabilidad, cuando el ahorro es igual o mayor al $7.23 \%$.

Las evaluaciones efectuadas sobre el consumo de petróleo y la consideración de un ahorro promedio anual del $30 \%$, nos garantizan un periodo de recuperación de la inversión ligeramente menor a dos años, tiempo que tiende a disminuir si se consideran los incrementos periódicos en los precios del petróleo.

\section{PROYECCCIONES}

La Tabla Nro. 1 - A indica el registro de consumo de petróleo en el departamento de Mantenimiento Campamentos para uso residencial en Cuajone (Villa Cuajone y Villa Botiflaca), para el año 1991.

TABLA $N^{\circ} 1-A$

\section{CONSUMO DE PETRÓLEO EN CAMPAMENTOS}

\begin{tabular}{|l|l|}
\hline MES & GALONES \\
\hline ENERO & 98724 \\
FEBRERO & 58585 \\
MARZO & 64558 \\
ABRIL & 78766 \\
MAYO & 60785 \\
JUNIO & 62403 \\
JULIO & 72769 \\
AGOSTO & 71666 \\
SETIEMBRE & 68765 \\
OCTUBRE & 64781 \\
NOVIEMBRE & 64646 \\
DECIEMBRE & 62630 \\
\hline TOTAL & $\mathbf{8 2 9 0 5 8}$ \\
\hline
\end{tabular}


TABLA $N^{\circ} 2-A$

INVERSIÓN EFECTUADA Y LUGARES DE APLICACIÓN

\begin{tabular}{|l|l|l|l|l|l|l|}
\hline ITEM & $N^{\circ}$ EQUIPO & LUGAR & $\begin{array}{l}\text { COSTO } \\
\text { SISTEMA } \\
\text { (US \$) }\end{array}$ & $\begin{array}{l}\text { CONSUMO } \\
\text { ANUAL } \\
\text { (GL) }\end{array}$ & $\begin{array}{l}\text { AHORRO } \\
\text { ANUAL } \\
\text { EST. (GAL) }\end{array}$ & $\begin{array}{l}\text { TIEMPO } \\
\text { RETORNO }\end{array}$ \\
\hline \multirow{2}{*}{2} & C1-301-348 & PABELLON & 25,200 & 38,750 & 11,627 & 1.8 \\
3 & C1-403-425 & PABELLON & 10,017 & 18,770 & 5,631 & 1.8 \\
4 & C1-CD13 & HOTEL & 3,339 & 5,121 & 1,536 & 1.8 \\
5 & C1-CD14 & PISCINA (*) & 16,200 & 1,462 & 10,800 & 1.8 \\
6 & C1-CD15 & C.E.P. AMERIC. & 3,339 & 1,075 & 500 & 1.8 \\
7 & CD9-50-73 & PABELLON & 7,000 & 11,700 & 3,510 & 1.8 \\
& CASAS V.C. & VILLA CUAJONE & 23,373 & 45,360 & 13,608 & 1.8 \\
\hline & B1-CD44 & EDIF. ADM & 4,500 & 5,383 & 1,614 & 1.8 \\
\hline
\end{tabular}

* El consumo de la piscina en 1991 estuvo limitado por decisiones de política interna, sin embargo, la tendencia de consumo anual es mayor.

La Tabla $\mathrm{N}^{\circ} 4$ - A resume el desarrollo de la inversión parcial efectuada y la proyección de aplicaciones en esta primera etapa, orientada al ahorro de petróleo en el calentamiento de agua (ítems 1, 2, 3, 4, 6 y 7) y calefacción ambiental (ítems 4 y 8 ).

En esta primera etapa, siendo el ahorro promedio de un $38 \%$, se expectó para los lugares de aplicación (Tabla 3-A), una disminución del consumo de petróleo del orden 48,826 galones. Comparativamente, este ahorro, en relación con el consumo registrado en 1991 (Tabla 3-A), el mismo que fue de 829,093 galones de petróleo, se estima del orden de 315,055 galones.

Esta proyección da indicadores claros de la viabilidad del proyecto y del considerable ahorro de energía fósil en campamentos mineros, contribuyendo al desarrollo del uso racional de la energía y a la disminución de los costos de producción al sector.

\section{SISTEMA DE COLECTORES SOLARES PARA UNA PISCINA (SGS-P)}

\section{Generalidades}

El sistema de colectores (140 m2 de área de locAción) instalado en las piscinas de Villa Cuajone y Toquepala, cubre las necesidades del atemperado de agua de las piscinas (180 m2, y una capacidad de 300 $\mathrm{m}^{3}$ ), en un rango de temperatura de $25-28{ }^{\circ} \mathrm{C}$. Estructualmente está constituido para absorbedores flexibles, hechos de polietileno (EPDM), de alta duración y resistencia a la radiación ultravioleta.

Este tipo de colectores posee diferentes tipos de configuraciones, habiéndose decidido la compra del denominado tipo "butterfly" (configuración mariposa), por la facilidad de su extensión sobre trayectos horizontales largos y por el hecho de que el uso de cabezales tipo "butterfly", reduce el descenso de presiones, al localizar los cabezales en el centro del colector, extendiéndose los colectores hacia ambos lados. La longitud máxima recomendada es de $24.5 \mathrm{~m}$.

En este caso, el sistema está constituido por ocho colectores de $18 \mathrm{~m} \times 1 \mathrm{~m}$. totalizando un área de aproximadamente $140 \mathrm{~m} 2$.

El agua fría ingresa por una tubería de 2" de diámetro por el centro del colector, distribuyéndose hacia ambos lados del mismo. El agua realiza una circulación (loop), transportando por transferencia el calor recepcionando en las placas colectoras y retornando a la tubería central de agua caliente.

El sistema ha sido instalado sobre el techo de la sala de filtros de la piscina y una extensión del terreno alrededor del cerro, que rodea la sala (Villa Cuajone), y para el caso de Toquepala, sobre dos plataformas inclinadas (fotos 1,2), diseñadas y coordinadas con el 
departamento de Ingeniería y Campamentos, en lo concerniente a su construcción.

\subsection{EL SISTEMA HIDRÁULICO}

El sistema hidráulico tradicional de una piscina posee los siguientes elementos: (figura $\mathrm{N}^{\circ} 8$ )

- Bombas de succión y circulación del agua de la piscina;

- Sistema de filtros que permiten eliminar impurezas en el agua de la piscina;

- Sistema de clarificación;

- Intercambiador de calor en conexión con una fuente tradicional de energía;

- Sistema automático de barrido y circulación del agua a lo largo y ancho de la piscina;

Equipo complementario de limpieza.

\subsection{EL SISTEMA DE CONTROL Y OPERACIÓN DEL SCSP}

El manejo y operación de una piscina requieren de técnicas y conocimientos que permitan, no sólo operar el sistema de filtros, sino que adicionalmente deben orientarse a mantener condiciones de salubridad, oxigenación y temperatura adecuadas en el agua de las piscinas. La instalación de un sistema solar para suplir y/o complementar el uso de petróleo (consumo promedio usual de $50 \mathrm{gls}$. al día), para mantener temperaturas de confort del orden de los $28-30^{\circ} \mathrm{C}$, ha requerido la realización de adecuaciones del sistema tradicional, no sólo debido a las necesidades propias de la instalación solar, sino también la interconexión con los procesos de limpieza de la piscina (sistema de filtros, uso de cloro, horas de operación, etc).

El sistema que controla el flujo de agua hacia los colectores es automático y funciona en combinación con sensores de temperatura, los mismos que sensan las temperaturas del agua de la piscina y la temperatura simulada del arreglo de colectores (Figura № 8). Es así que cuando existe una diferencia de temperaturas de 9 - $6{ }^{\circ} \mathrm{C}$ se activa la bomba y cuando la diferencia de temperatura es menor a $9-6^{\circ} \mathrm{C}$, la bomba se detiene. Ello permite una transferencia óptima de calor desde los colectores hacia el agua que circula por lo's mismos, en las horas de mayor insolación.

El sistema de control funciona con voltajes de $120 \mathrm{~V}$ a 220v AC y su salida es suficiente para hacer trabajar una motobomba hasta $1 \mathrm{Hp}$ con 120v AC ó 2 hp o más con 220 v AC. En este último caso debe adicionarse al circuito un relay externo $40^{\circ} \mathrm{C}$, de manera que cuando la temperatura correspondiente es alcanzada, la bomba de circulación se detiene.

\subsection{OPERACIÓN GENERAL DEL SISTEMA DE CALENTAMIENTO DE AGUA EN LA PISCINA DE VILLA CUAJONE}

El sistema de calentamiento de agua de la piscina en Cuajone y Toquepala, se divide en tres subsistemas:

1. Sistema de calor, mediante el uso de petróleo, utilizándose para ello un intercambiador de calor de contraflujo y un "boiler". Este sistema esta en "stand by", pudiendo utilizarse en periodos largos de nubosidad y/o falta de insolación, debiendo para ello abrirse las llaves de ingreso y salida del intercambiador y activarse el "boiler".

El modo de operación es conocido y es así como vino operando el sistema de calentamiento de la piscina.

El consumo de petróleo en promedio fue de 50 galones /día ó 1550 galones/mes.

2. El sistema de filtros ayuda a uniformizar o transportar el agua caliente a lo largo y ancho de la piscina y sistema de colectores.

3. El sistema solar descrito anteriormente y cuyas características de operación son como siguen: (Figuras 1 y 2)
a) Área total de colectores
$140 \mathrm{~m} 2$
b) Número de horas promedio de operación
$6 \mathrm{hrs} / \mathrm{día}$
c) Flujo
60 a 70 GPM
d) Incremento de temperatura
del agua a través de "cikectires": 6 hrs/dia
e) Masa total del agua circulante por día $21000 \mathrm{gls}$.

Generando un aporte energético de aproximadamente $900 \mathrm{kwh} /$ dia, o equivalente a 42 gls. de petróleo por día.

\subsubsection{Operación del sistema solar (Figura №8)}

El agua de la piscina es succionada por la bomba 1, haciendo que la misma pase a través de los filtros (presión 20 psi), y después de éstos se bifurque en dos líneas; una que pasa a través de la bomba 2, hacia los colectores (flujo 6070 GPM), y la otra que se desvía de regreso hacia la piscina.

El agua que va hacia los colectores se distribuye a 
través de un cabezal principal hacia ambos lados de los colectores, realizando una circulación de retorno invertido y recolectándose a través de un tubo cabezal de retorno (diametro 2"), para volver hacia la línea de regreso a la piscina.

En este proceso, tanto el flujo de agua caliente como el flujo derivado directamente a la piscina, se mezclan; repitiéndose el proceso mientras exista una diferencia de temperaturas de 6 a $9{ }^{\circ} \mathrm{C}$, entre la temperatura simulada de los colectores y la temperatura del agua que ingresa a la piscina. Esto último es regulado mediante el dispositivo de control descrito. Durante las horas de noche o cuando no existe suficiente incremento de temperaturas en el sistema de colectores, la bomba 2 para automáticamente, haciendo que el sistema drene. Este efecto se produce debido a la succión que se genera en la línea de retorno. El vacío generado es compensado mediante el ingreso de aire al sistema, a través de la válvula rompe vacío (vaccum breake valve).

A fin de mantener un flujo adecuado a través de los colectores, deben regularse las llaves de ingreso de agua a la bomba 2, así como la llave ubicada en la línea de bifurcación y retorno a la piscina.

Ello debe contrastarse con el flujómetro, el que debe marcar 60 - 70 GPM, cuando la bomba 2 está trabajando o indicar cero GPM, cuando la bomba 2 se detiene.

Es importante mantener presiones mínimas del orden
FIGURA $N^{\circ} 8$

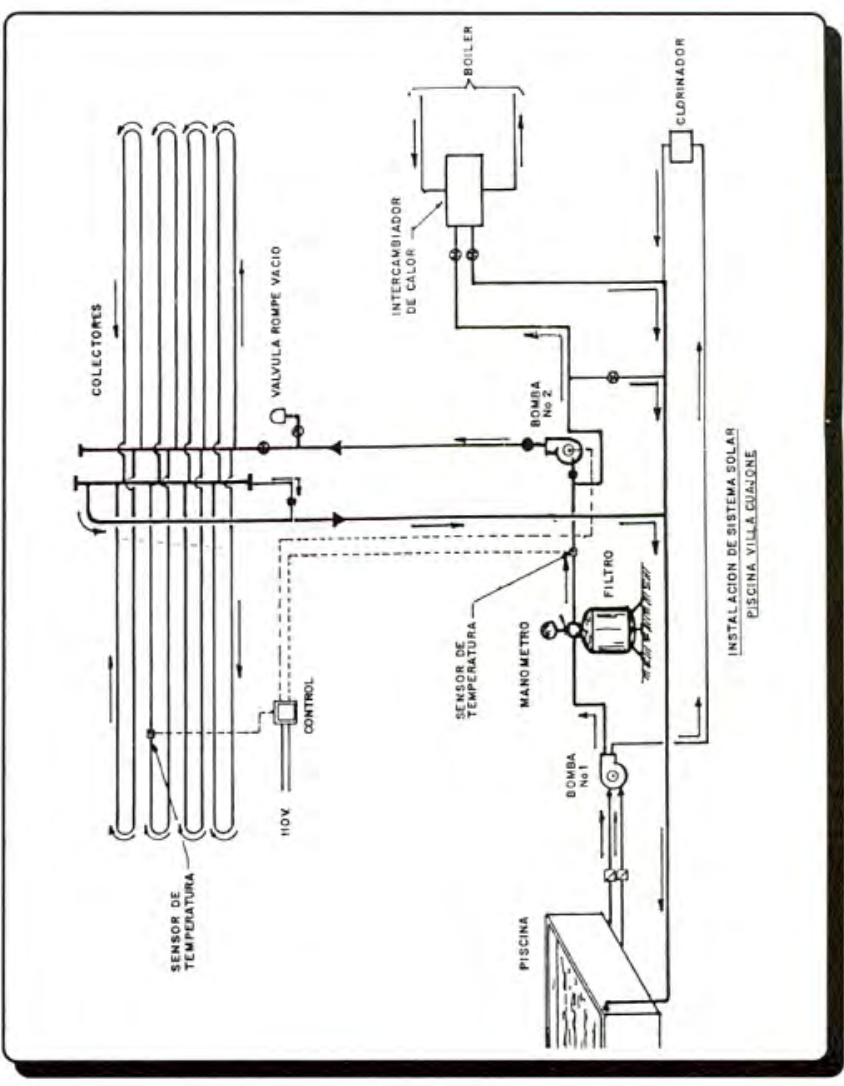

\section{SISTEMA COLECTOR SOLAR EN LA PISCINA DE TOQUEPALA (Fotos 1,2)}

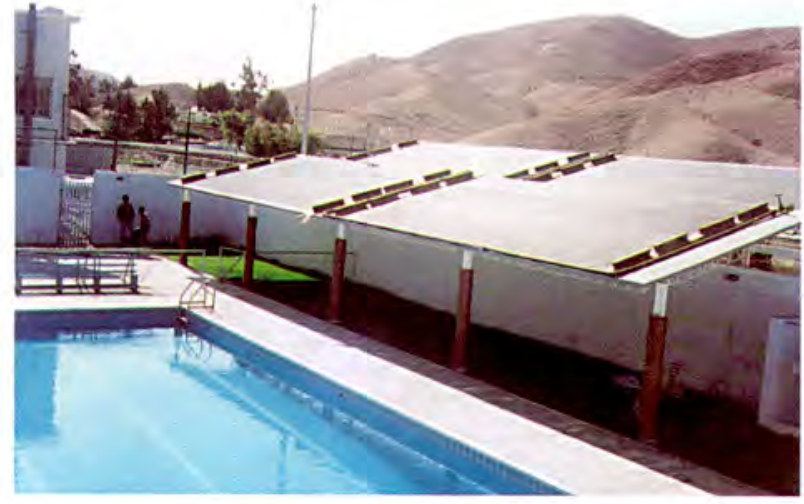

de 10 - 15 psi. (lectura de manómetro sobre la bomba 2), en el sistema solar, así como presiones del orden de 20 a 25 psi, en el filtro (lectura de manómetro del filtro). Si la presión en los filtros disminuye, hará que el flujo del agua a través de los colectores, también disminuya, en este caso es necesario seguir las indicaciones dadas en el manual de filtros.

\subsection{MANTENIMIENTO DEL SISTEMA SOLAR}

Los colectores solares requieren, de acuerdo a las condiciones de polvo en la atmósfera, una limpieza con agua y detergente diluido (poca cantidad) y dispersado sobre los colectores, debiendo después usarse una manguera con agua a presión para efectuar una mejor limpieza. Se han ubicado tres tomas de agua para este uso. Adicionalmente, se ha previsto la ubicación de dos

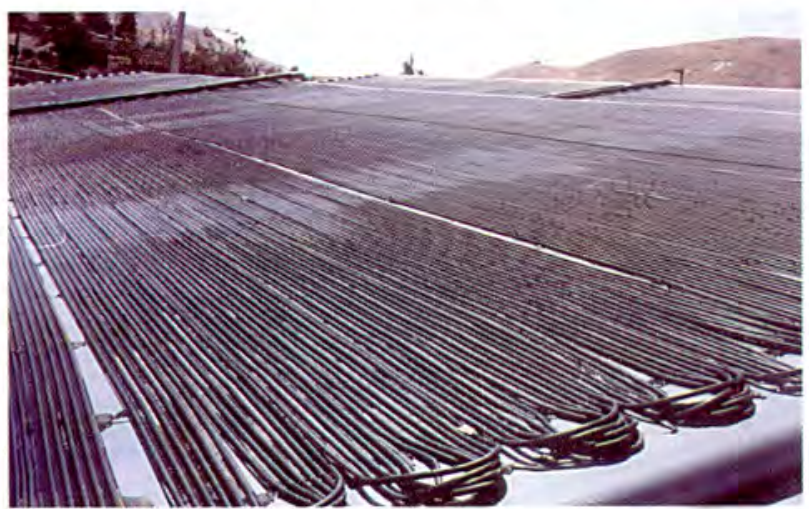


"strainer" (uno antes de la bomba 2 y después de la misma, justo antes de la subida del agua a los colectores), con la finalidad de retener probables fugas de arena o impurezas de los filtros hacia los colectores. Estos "strainers" deben chequearse sobre todo si se detectan descensos en la presión de los filtros.

El "vaccum breaker" debe también revisarse cada cierto tiempo. Un indicador de flujos intermitentes de agua hacia los colectores, es la variación en las lecturas y la fluctuación de la aguja del manómetro ubicado sobre la bomba 2. Una forma de controlar ello es regulando la llave de paso ubicada encima del "vaccum breaker" a la entrada del agua a los colectores.

\section{NOTAS BIBLIOGRÁFICAS}

Calefacción Solar para una Piscina en Toquepala -SPL. Reporte técnico M.Horn, C. Rivasplata, 1981.

Experimentos de Energía Solar. Reporte técnico, Div. Ingeniería SPCC - 1983.

Proyecto de Calefacción Solar en Viviendas (PCS). Reporte técnico UNJBG - SPCC/C.Rivasplata, 1986.

Informe 1 y 2 Proyecto PCS. Reporte técnico UNJBG - SPCC/ C.Rivasplata, 1987.

\section{APORTE Y AHORRO ENERGÉTICO DEL SISTEMA: TIEMPO DE RECUPERACIÓN DEL CAPITAL}

El sistema de calentamiento solar de la piscina de Villa Cuajone viene operando hace cuatro meses (setiembre 92. Figura 9), considerando las características de funcionamiento y las evaluaciones sobre el aporte energético solar entregado por el sistema y asumiendo una contribución solar del $83 \%$ (10 meses de insolación), se obtiene un ahorro de 42 galones/día ó 12,600 gls/año, con lo que se espera una recuperación del capital invertido en un tiempo aproximado de 13 meses.

Solar Heating Report. Reporte técnico, C.Rivasplata, 1991 1992.

Swimming Pool Heating by Solar Energy in Cuajone. Reporte técnico, C.Rivasplata, 1992.

How to Design and Build a Soilar Swimming Heater. Francis de Winter, 1985.

Factibilidad Económica de Proyectos de Inversión de Energía. H.Korber y G. Schuz, basado en H. Finck y G. OelestGTZ.

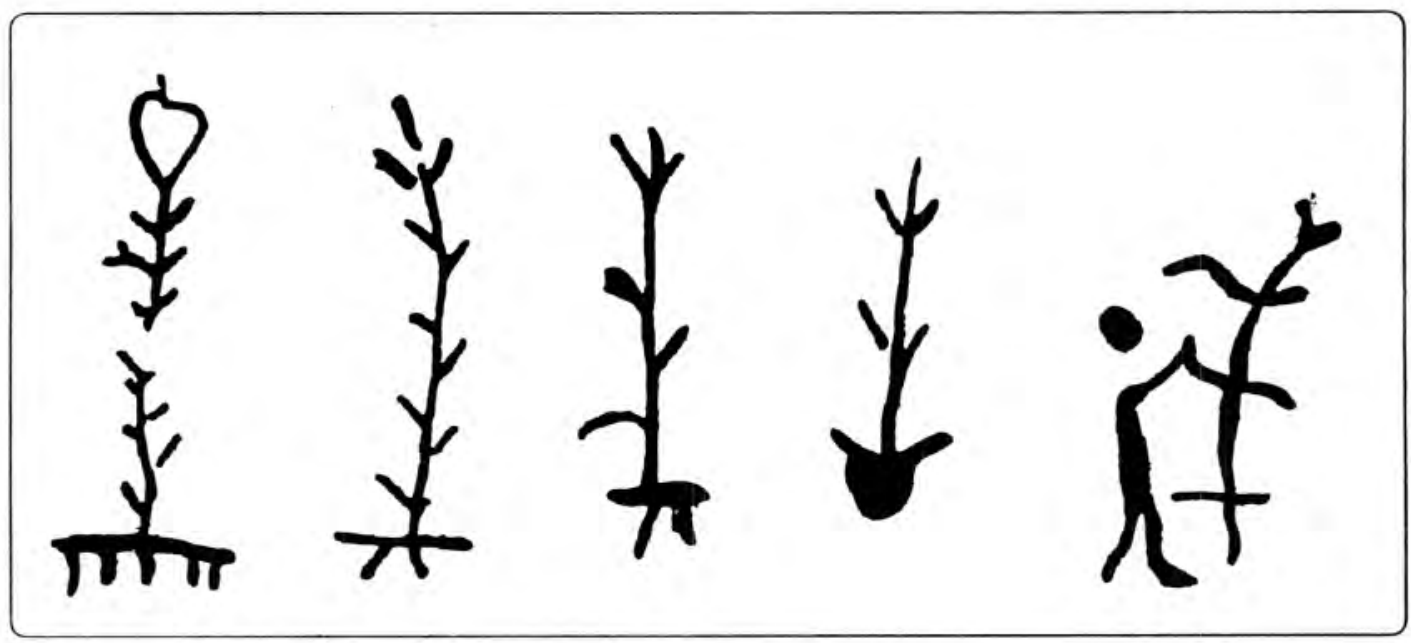

Tallos y raices 